\title{
Ccreative
commons
}

ISSN 1855-3966 (printed edn.), ISSN 1855-3974 (electronic edn.)

ARS MATHEMATICA CONTEMPORANEA 22 (2022) \#P3.10

https://doi.org/10.26493/1855-3974.2308.de6

(Also available at http://amc-journal.eu)

\section{The (non-)existence of perfect codes in Lucas cubes}

\author{
Michel Mollard \\ Institut Fourier, CNRS, Université Grenoble Alpes, France
}

Received 10 April 2020, accepted 10 December 2021, published online 9 June 2022

\begin{abstract}
The Fibonacci cube of dimension $n$, denoted as $\Gamma_{n}$, is the subgraph of the $n$-cube $Q_{n}$ induced by vertices with no consecutive 1's. Ashrafi and his co-authors proved the nonexistence of perfect codes in $\Gamma_{n}$ for $n \geq 4$. As an open problem the authors suggest to consider the existence of perfect codes in generalizations of Fibonacci cubes. The most direct generalization is the family $\Gamma_{n}\left(1^{s}\right)$ of subgraphs induced by strings without $1^{s}$ as a substring where $s \geq 2$ is a given integer. In a precedent work we proved the existence of a perfect code in $\Gamma_{n}\left(1^{s}\right)$ for $n=2^{p}-1$ and $s \geq 3.2^{p-2}$ for any integer $p \geq 2$. The Lucas cube $\Lambda_{n}$ is obtained from $\Gamma_{n}$ by removing vertices that start and end with 1 . Very often the same problems are studied on Fibonacci cubes and Lucas cube. In this note we prove the non-existence of perfect codes in $\Lambda_{n}$ for $n \geq 4$ and prove the existence of perfect codes in some generalized Lucas cube $\Lambda_{n}\left(1^{s}\right)$.
\end{abstract}

Keywords: Error correcting codes, perfect code, Fibonacci cube.

Math. Subj. Class. (2020): 94B50, 05C69

\section{Introduction and notations}

An interconnection topology can be represented by a graph $G=(V, E)$, where $V$ denotes the processors and $E$ the communication links. The hypercube $Q_{n}$ is a popular interconnection network because of its structural properties.

The Fibonacci cube was introduced in [8] as a new interconnection network. This graph is an isometric subgraph of the hypercube which is inspired in the Fibonacci numbers. It has attractive recurrent structures such as its decomposition into two subgraphs which are also Fibonacci cubes by themselves. Structural properties of these graphs were more extensively studied afterwards. See [12] for a survey.

E-mail address: michel.mollard@univ-grenoble-alpes.fr (Michel Mollard) 
Lucas cubes, introduced in [17], have attracted the attention as well due to the fact that these cubes are closely related to the Fibonacci cubes. They have also been widely studied $[3,4,6,11,13,14,18,20,23]$.

We will next define some concepts needed in this paper. Let $G$ be a connected graph. The open neighbourhood of a vertex $u$ is $N_{G}(u)$ the set of vertices adjacent to $u$. The closed neighbourhood of $u$ is $N_{G}[u]=N_{G}(u) \cup\{u\}$. The distance between two vertices denoted $d_{G}(x, y)$ is the length of a shortest path between $x$ and $y$. We have thus $N_{G}[u]=$ $\left\{v \in V(G) ; d_{G}(u, v) \leq 1\right\}$. We will use the notations $d(x, y)$ and $N[u]$ when the graph is unambiguous.

A dominating set $D$ of $G$ is a set of vertices such that every vertex of $G$ belongs to the closed neighbourhood of at least one vertex of $D$. In [2], Biggs initiated the study of perfect codes in graphs as a generalization of classical 1-error perfect correcting codes. A code $C$ in $G$ is a set of vertices $C$ such that for every pair of distinct vertices $c, c^{\prime}$ of $C$ we have $N_{G}[c] \cap N_{G}\left[c^{\prime}\right]=\emptyset$ or equivalently such that $d_{G}\left(c, c^{\prime}\right) \geq 3$.

A perfect code of a graph $G$ is both a dominating set and a code. It is thus a set of vertices $C$ such that every vertex of $G$ belongs to the closed neighbourhood of exactly one vertex of $C$. A perfect code is also known as an efficient dominating set. The existence or non-existence of perfect codes have been considered for many graphs. See the introduction of [1] for some references.

The vertex set of the $n$-cube $Q_{n}$ is the set $\mathbb{B}_{n}$ of binary strings of length $n$, two vertices being adjacent if they differ in precisely one position. Classical 1-error correcting codes and perfect codes are codes and perfect codes in the graph $Q_{n}$. The weight of a binary string is the number of 1 s. The concatenation of strings $\boldsymbol{x}$ and $\boldsymbol{y}$ is denoted $\boldsymbol{x} \| \boldsymbol{y}$ or just $\boldsymbol{x} \boldsymbol{y}$ when there is no ambiguity. A string $\boldsymbol{f}$ is a substring of a string $s$ if there exist strings $\boldsymbol{x}$ and $\boldsymbol{y}$, may be empty, such that $s=\boldsymbol{x} \boldsymbol{f} \boldsymbol{y}$.

A Fibonacci string of length $n$ is a binary string $\boldsymbol{b}=b_{1} \ldots b_{n}$ with $b_{i} \cdot b_{i+1}=0$ for $1 \leq i<n$. In other words a Fibonacci string is a binary string without 11 as substring. The Fibonacci cube $\Gamma_{n}(n \geq 1)$ is the subgraph of $Q_{n}$ induced by the Fibonacci strings of length $n$. Adjacent vertices in $\Gamma_{n}$ differ in one bit. Because of the empty string, $\Gamma_{0}=K_{1}$.

A Fibonacci string of length $n$ is a Lucas string if $b_{1} \cdot b_{n} \neq 1$. That is, a Lucas string has no two consecutive 1's including the first and the last elements of the string. The Lucas cube $\Lambda_{n}$ is the subgraph of $Q_{n}$ induced by the Lucas strings of length $n$. We have $\Lambda_{0}=\Lambda_{1}=K_{1}$.

Let $\mathcal{F}_{n}$ and $\mathcal{L}_{n}$ be the set of strings of Fibonacci strings and Lucas strings of length $n$.

By $\Gamma_{n, k}$ and $\Lambda_{n, k}$ we denote the vertices of of weight $k$ in respectively $\Gamma_{n}$ and $\Lambda_{n}$.

Since

$$
\mathcal{L}_{n}=\left\{0 s ; s \in \mathcal{F}_{n-1}\right\} \cup\left\{10 s 0 ; s \in \mathcal{F}_{n-3}\right\}
$$

and

$$
\left|\Gamma_{n, k}\right|=\left(\begin{array}{c}
n-k+1 \\
k
\end{array}\right)
$$

it is immediate to derive the following classical result.

Proposition 1.1. Let $n \geq 1$. The number of vertices of weight $k \leq n$ in $\Lambda_{n}$ is

$$
\left|\Lambda_{n, k}\right|=\left(\begin{array}{c}
n-k \\
k
\end{array}\right)+\left(\begin{array}{c}
n-k-1 \\
k-1
\end{array}\right) .
$$



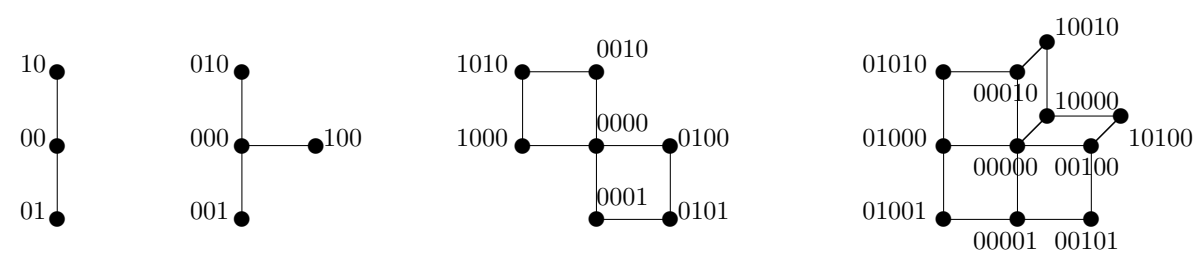

Figure 1: $\Gamma_{2}=\Lambda_{2}, \Lambda_{3}, \Lambda_{4}$ and $\Lambda_{5}$.

It will be convenient to consider the binary strings of length $n$ as vectors of $\mathbb{F}^{n}$ the vector space of dimension $n$ over the field $\mathbb{F}=\mathbb{Z}_{2}$ thus to associate to a string $x_{1} x_{2} \ldots x_{n}$ the vector $\theta\left(x_{1} x_{2} \ldots x_{n}\right)=\left(x_{1}, x_{2}, \ldots, x_{n}\right)$. The Hamming distance between two vectors $\boldsymbol{x}, \boldsymbol{y} \in \mathbb{F}^{n}, d(\boldsymbol{x}, \boldsymbol{y})$ is the number of coordinates in which they differ. By the correspondence $\theta$ we can define the binary sum $\boldsymbol{x}+\boldsymbol{y}$ and the Hamming distance $d(\boldsymbol{x}, \boldsymbol{y})$ of strings in $\mathbb{B}_{n}$. Note that the Hamming distance is the usual graph distance in $Q_{n}$.

We will first recall some basic results about perfect codes in $Q_{n}$. Since $Q_{n}$ is a regular graph of degree $n$ the existence of a perfect code of cardinality $|C|$ implies $|C|(n+1)=2^{n}$ thus a necessary condition of existence is that $n+1$ is a power of 2 thus that $n=2^{p}-1$ for some integer $p$.

For any integer $p$ Hamming [7] constructed, a linear subspace of $\mathbb{F}^{2^{p}-1}$ which is a perfect code. It is easy to prove that all linear perfect codes are Hamming codes. Notice that $1^{n}$ belongs to the Hamming code of length $n$.

In 1961 Vasilev [22], and later many authors, see [5, 21] for a survey, constructed perfect codes which are not linear codes.

In a recent work [1] Ashrafi and his co-authors proved the non-existence of perfect codes in $\Gamma_{n}$ for $n \geq 4$. As an open problem the authors suggest to consider the existence of perfect codes in generalizations of Fibonacci cubes. The most complete generalization proposed in [9] is, for a given string $\boldsymbol{f}$, to consider $\Gamma_{n}(\boldsymbol{f})$ the subgraph of $Q_{n}$ induced by strings that do not contain $f$ as substring. Since Fibonacci cubes are $\Gamma_{n}(11)$ the most immediate generalization $[15,19]$ is to consider $\Gamma_{n}\left(1^{s}\right)$ for a given integer $s$. In [16] we proved the existence of a perfect code in $\Gamma_{n}\left(1^{s}\right)$ for $n=2^{p}-1$ and $s \geq 3.2^{p-2}$ for any integer $p \geq 2$.

In the next section we will prove the main result of this note.

Theorem 1.2. The Lucas cube $\Lambda_{n}, n \geq 0$, admits a perfect code if and only if $n \leq 3$.

\section{Perfect codes in Lucas cube}

It can be easily checked by hand that $\left\{0^{n}\right\}$ is a perfect code of $\Lambda_{n}$ for $n \leq 3$ and that $\Lambda_{4}$ and $\Lambda_{5}$ do not contain a perfect code (Figure 1).

Assume thus $n \geq 6$.

Note first that from Proposition 1.1 we have

$$
\left|\Lambda_{n, 2}\right|=\frac{n(n-3)}{2} \text { and }\left|\Lambda_{n, 3}\right|=\frac{n(n-4)(n-5)}{6} .
$$

Therefore $\Lambda_{n, 2}$ and $\Lambda_{n, 3}$ are none empty. 
Let $\Lambda_{n, k}^{1}$ be the vertices of $\Lambda_{n, k}$ that start with 1 . Since $\mathcal{L}_{n}=\left\{0 s ; s \in \mathcal{F}_{n-1}\right\} \cup$ $\left\{10 s 0 ; s \in \mathcal{F}_{n-3}\right\}$ the number of vertices in $\Lambda_{n, k}^{1}$ is

$$
\left|\Lambda_{n, k}^{1}\right|=\left|\Gamma_{n-3, k-1}\right|=\left(\begin{array}{c}
n-1-k \\
k-1
\end{array}\right) .
$$

Lemma 2.1. If $n \geq 6$ and $C$ is a perfect code of $\Lambda_{n}$ then $0^{n} \in C$.

Proof. Suppose on the contrary that $0^{n} \notin C$. Since $0^{n}$ must be dominated there exists a vertex in $\Lambda_{n, 1} \cap C$. This vertex is unique and because of the circular symmetry of $\Lambda_{n}$ we can assume $10^{n-1} \in C$.

Since $0^{n} \notin C$ the other vertices of $\Lambda_{n, 1}$ must be dominated by vertices in $\Lambda_{n, 2}$. But a vertex in $\Lambda_{n, 2}$ has precisely two neighbors in $\Lambda_{n, 1}$ thus $n$ must be odd and

$$
\left|\Lambda_{n, 2} \cap C\right|=\frac{n-1}{2} .
$$

The unique vertex $10^{n-1}$ in $\Lambda_{n, 1} \cap C$ has exactly $n-3$ neighbors in $\Lambda_{n, 2}$. Let $D$ be the vertices of $\Lambda_{n, 2}$ not in $C$ and not dominated by $10^{n-1}$. Vertices in $D$ must be dominated by vertices in $\Lambda_{n, 3} \cap C$. Each vertex of $\Lambda_{n, 3} \cap C$ has exactly exacty three neighbors in $\Lambda_{n, 2}$. Thus 3 divides the number of vertices in $D$. This number is

$$
|D|=\left|\Lambda_{n, 2}\right|-(n-3)-\frac{n-1}{2}=\frac{n^{2}-6 n+7}{2} .
$$

This is not possible since there exists no odd integer $n$ such that 6 divides $n^{2}+1$. Indeed since $n$ is odd, 6 does not divide $n$ thus divides $(n+1)(n-1)=n^{2}-1$ or $(n+2)(n-2)=$ $n^{2}-4$ or $(n+3)(n-3)=n^{2}-9$ thus cannot divide $n^{2}+1$.

We are now going to prove Theorem 1.2.

Proof of Theorem 1.2. Let $n \geq 6$ and $C$ be a perfect code. Since $0^{n} \in C$ all vertices of $\Lambda_{n, 1}$ are dominated by $0^{n}$ and thus $\Lambda_{n, 2} \cap C=\Lambda_{n, 1} \cap C=\emptyset$. Consequently, each vertex of $\Lambda_{n, 2}$ must be dominated by a vertex in $\Lambda_{n, 3}$. Since each vertex in $\Lambda_{n, 3}$ has precisely three neighbors in $\Lambda_{n, 2}$ we obtain that

$$
\left|\Lambda_{n, 3} \cap C\right|=\frac{\left|\Lambda_{n, 2}\right|}{3} .
$$

This number must be an integer thus 3 divides $\left|\Lambda_{n, 2}\right|=\frac{n(n-3)}{2}$ and therefore 3 divides $n(n-3)$. This is only possible if $n$ is a multiple of 3 .

Each vertex of $\Lambda_{n, 2}^{1}$ must be dominated by a vertex in $\Lambda_{n, 3}^{1}$. Furthermore a vertex in $\Lambda_{n, 3}^{1}$ has precisely two neighbors in $\Lambda_{n, 2}^{1}$. Therefore $\left|\Lambda_{n, 2}^{1}\right|=n-3$ must be even and thus $n=6 p+3$ for some integer $p \geq 1$.

Let $E$ be the set of vertices of $\Lambda_{n, 3}$ not in $C$. Vertices in $E$ must be dominated by a vertex in $\Lambda_{n, 4}$. Furthermore each vertex in $\Lambda_{n, 4}$ has precisely four neighbors in $\Lambda_{n, 3}$.

Therefore 4 divides $|E|$ with

$$
|E|=\left|\Lambda_{n, 3}\right|-\left|\Lambda_{n, 3} \cap C\right|=\frac{n(n-4)(n-5)}{6}-\frac{n(n-3)}{6}=\frac{n\left(n^{2}-10 n+23\right)}{6} .
$$

Replacing $n$ by $6 p+3$ we obtain that 4 divides the odd number $(2 p+1)\left(18 p^{2}-12 p+1\right)$. This contradiction proves Theorem 1.2. 


\section{Perfect codes in generalized Lucas cube}

The analogous of the generalisation of Fibonacci cube $\Gamma_{n}\left(1^{s}\right)$ for Lucas cube is the family $\Lambda_{n}\left(1^{s}\right)$ of subgraphs of $Q_{n}$ induced by strings without $1^{s}$ as a substring in a circular manner where $s \geq 2$ is a given integer. More formally [10] for any binary strings $b_{1} b_{2} \ldots b_{n}$ and each $1 \leq i \leq n$, call $b_{i} b_{i+1} \ldots b_{n} b_{1} \ldots b_{i-1}$ the $i$-th circulation of $b_{1} b_{2} \ldots b_{n}$. The generalized Lucas cube $\Lambda_{n}\left(1^{s}\right)$ is the subgraph of $Q_{n}$ induced by strings without a circulation containing $1^{s}$ as a substring.

In [16] the existence of a perfect code in $\Gamma_{n}\left(1^{s}\right)$ is proved for $n=2^{p}-1$ and $s \geq 3.2^{p-2}$ for any integer $p \geq 2$.

The strategy used in this construction is to build a perfect code $C$ in $Q_{n}$ such that no vertex of $C$ contains $1^{s}$ as substring. The set $C$ is also a perfect code in $\Gamma_{n}\left(1^{s}\right)$ since each vertex of $\Gamma_{n}\left(1^{s}\right)$ belongs to the unique closed neighbourhood in $Q_{n}$ thus in $\Gamma_{n}\left(1^{s}\right)$ of a vertex in $C$. Because of the following proposition we cannot use the same idea for $\Lambda_{n}\left(1^{s}\right)$ and $s \leq n-1$.

Proposition 3.1. Let $n$ be an integer and $2 \leq s \leq n-1$. If $C$ is a perfect code in $Q_{n}$ then some word of $C$ contains a circulation of $1^{s}$ as a substring.

Proof. Let $C$ be a such a perfect code in $Q_{n}$ then $1^{n} \notin C$. Thus $1^{n}$ must be neighbour of a vertex $c$ in $C$. Since $c=1^{i} 01^{n-1-i}$ for some integer $i$ the $i+1$ th-circulation of $c$ is $1^{n-1} 0$.

We can supplement this proposition by the two following results.

Proposition 3.2. Let $p \geq 2$ and $n=2^{p}-1$ then there exists a perfect code in $\Lambda_{n}\left(1^{n}\right)$ of order $|C|=\frac{2^{n}}{n+1}$.

Proof. Let $D$ be a Hamming code of length $n$ and $C=\left\{d+\left(0^{n-1} 1\right) ; d \in D\right\}$. Since $1^{n} \in D$, the set $C$ is a perfect code of $Q_{n}$ such that $1^{n} \notin C$. Since $\Lambda_{n}\left(1^{n}\right)$ is obtained from $Q_{n}$ by the deletion of $1^{n}$ every vertex of $\Lambda_{n}\left(1^{n}\right)$ is in the closed neighbourhood of exactly one vertex of $C$.

Proposition 3.3. Let $p \geq 2$ and $n=2^{p}-1$ then there exists a perfect code in $\Lambda_{n}\left(1^{n-1}\right)$ and in $\Lambda_{n}\left(1^{n-2}\right)$ of order $|C|=\frac{2^{n}}{n+1}-1$.

Proof. Let $D$ be a Hamming code of length $n$. Then $D$ is a perfect code of $Q_{n}$ such that $1^{n} \in D$. Since $\Lambda_{n}\left(1^{n-1}\right)$ is obtained from $Q_{n}$ by the deletion of the closed neighbourhood of $1^{n}$ every vertex of $\Lambda_{n}\left(1^{n-1}\right)$ is in the closed neighbourhood of exactly one vertex of $C=D-\left\{1^{n}\right\}$. Furthermore since $1^{n} \in D$ there is no vertex of weight $n-2$ in $D$. Let $u$ be a vertex of $\Lambda_{n}\left(1^{n-2}\right)$ and $f(u)$ be the vertex in $D$ such that $u \in N_{Q^{n}}[u]$. Since there is no vertex in $D$ with weight $n-1$ or $n-2$ there is no circulation of $f(u)$ containing $1^{n-2}$ as a substring. Therefore $f(u)$ is a vertex of $\Lambda_{n}\left(1^{n-2}\right)$ and $u \in N_{\Lambda_{n}\left(1^{n-2}\right)}[f(u)]$. Since a code in $Q_{n}$ is a code in each of its subgraph $C$ is a perfect code of $\Lambda_{n}\left(1^{n-2}\right)$.

\section{ORCID iDs}

Michel Mollard (D) https://orcid.org/0000-0002-3602-8019 


\section{References}

[1] A. R. Ashrafi, J. Azarija, A. Babai, K. Fathalikhani and S. Klavžar, The (non-) existence of perfect codes in Fibonacci cubes, Inf. Process. Lett. 116 (2016), 387-390, doi:10.1016/j.ipl. 2016.01.010.

[2] N. Biggs, Perfect codes in graphs, J. Comb. Theory, Ser. B 15 (1973), 289-296, doi:10.1016/ 0095-8956(73)90042-7.

[3] A. Castro, S. Klavžar, M. Mollard and Y. Rho, On the domination number and the 2-packing number of Fibonacci cubes and Lucas cubes, Comput. Math. Appl. 61 (2011), 2655-2660, doi:10.1016/j.camwa.2011.03.012.

[4] A. Castro and M. Mollard, The eccentricity sequences of Fibonacci and Lucas cubes, Discrete Math. 312 (2012), 1025-1037, doi:10.1016/j.disc.2011.11.006.

[5] G. Cohen, I. Honkala, S. Litsyn and A. Lobstein, Covering Codes, volume 54 of North-Holland Math. Libr., Elsevier, Amsterdam, 1997, doi:10.1016/s0924-6509(97)x8001-8.

[6] E. Dedó, D. Torri and N. Zagaglia Salvi, The observability of the Fibonacci and the Lucas cubes, Discrete Math. 255 (2002), 55-63, doi:10.1016/s0012-365x(01)00387-9.

[7] R. W. Hamming, Error detecting and error correcting codes, Bell Syst. Tech. J. 29 (1950), 147160, doi:10.1002/j.1538-7305.1950.tb00463.x.

[8] W.-J. Hsu, Fibonacci cubes-a new interconnection topology, IEEE Transactions on Parallel and Distributed Systems 4 (1993), 3-12, doi:10.1109/71.205649.

[9] A. Ilić, S. Klavžar and Y. Rho, Generalized Fibonacci cubes, Discrete Math. 312 (2012), 2-11, doi:10.1016/j.disc.2011.02.015.

[10] A. Ilić, S. Klavžar and Y. Rho, Generalized Lucas cubes, Appl. Anal. Discrete Math. 6 (2012), 82-94, doi:10.2298/aadm120108002i.

[11] A. Ilić and M. Milošević, The parameters of Fibonacci and Lucas cubes, Ars Math. Contemp. 12 (2017), 25-29, doi:10.26493/1855-3974.915.f48.

[12] S. Klavžar, Structure of Fibonacci cubes: a survey, J. Comb. Optim. 25 (2013), 505-522, doi: 10.1007/s10878-011-9433-z.

[13] S. Klavžar and M. Mollard, Cube polynomial of Fibonacci and Lucas cubes, Acta Appl. Math. 117 (2012), 93-105, doi:10.1007/s10440-011-9652-4.

[14] S. Klavžar, M. Mollard and M. Petkovšek, The degree sequence of Fibonacci and Lucas cubes, Discrete Math. 311 (2011), 1310-1322, doi:10.1016/j.disc.2011.03.019.

[15] J. Liu and W.-J. Hsu, Distributed algorithms for shortest-path, deadlock-free routing and broadcasting in a class of interconnection topologies, in: Proceedings Sixth International Parallel Processing Symposium, 1992 pp. 589-596, doi:10.1109/ipps.1992.222963.

[16] M. Mollard, The existence of perfect codes in a family of generalized Fibonacci cubes, Inf. Process. Lett. 140 (2018), 1-3, doi:10.1016/j.ipl.2018.07.010.

[17] E. Munarini, C. Perelli Cippo and N. Salvi, On the Lucas cubes, Fibonacci Q. 39 (2001), https://www.fq.math.ca/39-1.html.

[18] M. Ramras, Congestion-free routing of linear permutations on Fibonacci and Lucas cubes, Australas. J. Comb. 60 (2014), 1-10, https : / / a jc . maths . uq. edu . au/ ?page=get_ volumes\&volume $=60$.

[19] N. Z. Salvi, On the existence of cycles of every even length on generalized Fibonacci cubes, Matematiche 51 (1996), 241-251, https: / / lematematiche.dmi . unict.it/ index.php/lematematiche/article/view/475. 
[20] E. Saygi and Ö. Eğecioğlu, q-counting hypercubes in Lucas cubes, Turk. J. Math. 42 (2018), 190-203, doi:10.3906/mat-1605-2.

[21] F. I. Solov'eva, On perfect binary codes, in: General theory of information transfer and combinatorics, Elsevier, Amsterdam, pp. 371-372, 2005, doi:10.1016/j.endm.2005.07.059.

[22] Y. Vasil'ev, On nongroup close-packed codes, Probl. Kibern. 8 (1962), 337-339.

[23] X. Wang, X. Zhao and H. Yao, Structure and enumeration results of matchable Lucas cubes, Discrete Appl. Math. 277 (2020), 263-279, doi:10.1016/j.dam.2019.09.011. 